\title{
NEW RESULTS ON BANDWIDTH OF GaAs PIN PHOTODIODES
}

\author{
M.A. GRADO CAFFARO \\ consultant, Madrid, Spain \\ M. GRADO CAFFARO \\ CSIC, Madrid, Spain \\ (Received March 3, 1993; in final form March 31, 1993)
}

In this paper an equivalent circuit of a GaAs PIN photodiode for the millimeter wave range is proposed. Moreover, an approximate expression for the bandwidth of this circuit is obtained. By using the effective quality factor concept, an equation that includes the value of a hypothetical resistance involved in the circuit mentioned above, is derived. In the context of fiber optic communications, this "resistance" represents the losses due to the fiber-photodiode coupling.

\section{INTRODUCTION}

The excellent behavior of GaAs PIN photodiodes for higher frequencies than a few $\mathrm{GHz}$ is well-known. In particular, the combination GaAs PIN photodiodeHEMT constitutes a good solution in order to choose an adequate hybrid receiver for fiber optic communications. Also, GaAs PIN photodiode-GaAs MESFET is an excellent photodetector-amplifier.

On the other hand, it is well-known that the bandwidth of a generalized PIN photodiode is limited by the following factors:

1) Transit time of generated electron-hole pairs in the depletion zone.

2) Active region capacitance.

3) Diffusion of slow moving carriers generated outside the active zone.

4) Stray effects due to the connection of the device to an external circuit.

In practice, the speed of response of the device is governed by the finite transient time of electron-hole pairs generated in the depletion region, and by the time constant related to the behavior of the device as a parallel plate capacitor ${ }^{1.2}$.

According to Wang and Bloom ${ }^{3}$, the analysis of an equivalent circuit of the photodetector in question is feasible. From this circuit it is easy to obtain an approximate expression that gives the bandwidth of the circuit. In addition, the consideration of an extension of this circuit is feasible. 


\section{CALCULATIONS}

First, in accordance with an experimental work of Wang and Bloom ${ }^{3}$, we propose the following equivalent circuit (fig. 1) for the detector in question.

Wang and Bloom introduced a series coil of $50 \mathrm{pH}$ in order to maximize the speed of response (or bandwidth) of the circuit represented in figure 1 . In this circuit, $C$ is the junction capacitance of the device and $R$ represents the load resistance of the photodetector. Bandwidth is controlled by the time constants $\tau_{\mathrm{tr}}$ and $\tau_{\mathrm{RC}} ; \tau_{\mathrm{tr}}$ is a measure of the finite transit time of electron-hole pairs generated in the depletion zone; $\tau_{\mathrm{RC}}$ results from considering the device as a parallel plate capacitor. Then, the effective carrier life-time of the electron-hole pairs is given by the following approximate expression

$$
\tau_{\mathrm{cff}} \cong 3 \tau_{\mathrm{tr}}+2 \tau_{\mathrm{RC}}
$$

The above equation results from our research work in conjunction with estimations of other researchers ${ }^{3-5}$. Consequently, we have

$$
\mathrm{B}=\frac{1}{\tau_{\mathrm{eff}}}=\frac{1}{3 \tau_{\mathrm{tr}}+2 \tau_{\mathrm{RC}}}
$$

where $B$ is the $-3 \mathrm{~dB}$ bandwidth without the introduction of the inductance. Now, denoting by $\bar{B}$ the $-3 \mathrm{~dB}$ bandwidth including this inductance, it is trivial that the new $-3 \mathrm{~dB}$ bandwidth is given by

$$
\overline{\mathrm{B}}=\frac{f_{0}}{\mathrm{Q}}
$$

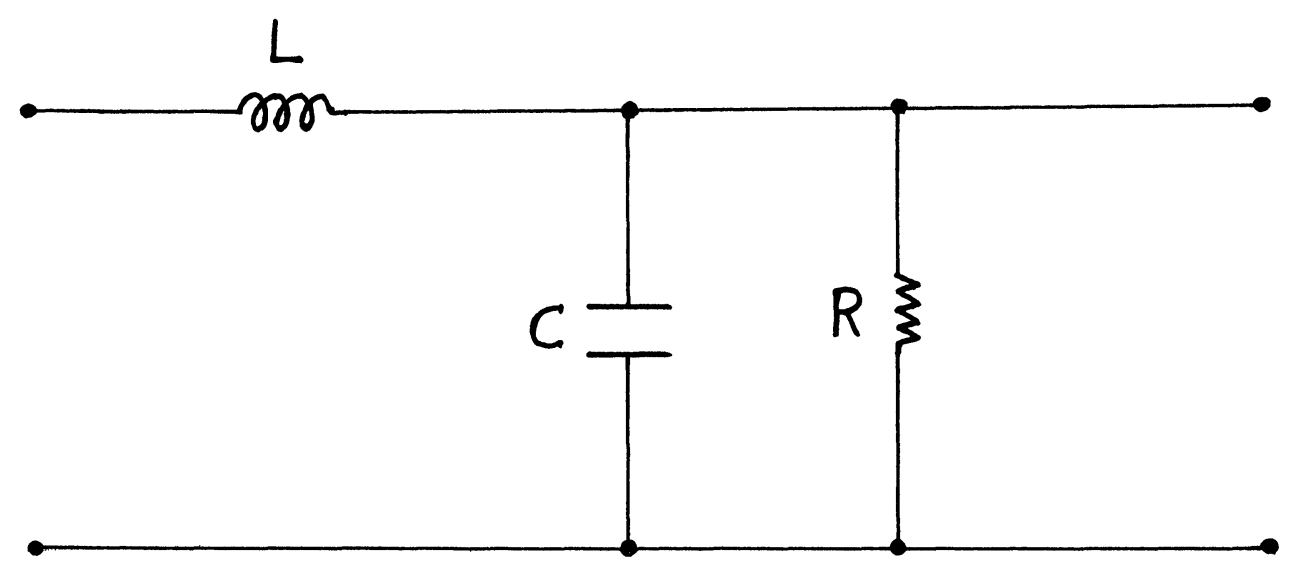

FIGURE 1 Equivalent circuit of the detector with a series coil. 
where $f_{0}$ is the resonant frequency and $\mathrm{Q}$ the quality factor. In particular, we have

$f_{0}=\frac{1}{2 \pi \sqrt{\mathrm{LC}}}$

For a $5 \mu \mathrm{m}$ square GaAs PIN photodiode with a $0.40 \mu \mathrm{m}$ active zone we have established the following values: $\tau_{\mathrm{tr}} \cong 4 \mathrm{ps}, \tau_{\mathrm{RC}} \cong 0.6 \mathrm{ps}$. From these values, we can consider approximately $\tau_{\mathrm{tr}} \cong 7 \tau_{\mathrm{RC}}$. Moreover, from Eqn. (2) we get

$\mathrm{B}<\frac{1}{5 \tau_{\mathrm{RC}}^{\prime}}$

Next, we shall obtain a useful approximate expression for B by virtue of the assumption $\tau_{\mathrm{tr}} \cong 7 \tau_{\mathrm{RC}}$. To get this end, we shall use a second order Taylor-Mc. Laurin expansion (considering small $\tau_{\mathrm{RC}} / \tau_{\mathrm{tr}}$ ) and the function

$\mathrm{B}=\frac{1}{\tau_{\mathrm{tr}}\left(3+2 \cdot \frac{\tau_{\mathrm{RC}}}{\tau_{\mathrm{tr}}}\right)}$

Then we obtain

$\mathrm{B} \cong \frac{1}{3 \tau_{\mathrm{tr}}}\left[\left(\frac{2 \tau_{\mathrm{RC}}}{3 \tau_{\mathrm{tr}}}-1\right)^{2}+\frac{2 \tau_{\mathrm{RC}}}{3 \tau_{\mathrm{tr}}}\right]$

Although equation (6) is slightly more complicated than (2), it is easy to observe the usefulness of (6) for the majority of GaAs PIN photodiodes.

Finally, we shall study the following situation: the circuit of fig. 1 launched by a hypothetical microwave generator with internal resistance $R_{g}$. We consider photodiodes launched by optical power belonging to the far infrared range so that the simulation of the optical excitation by means of a millimeter-wave generator is feasible; we assume a $250-300 \mathrm{GHz}$-millimeter wave generator. Thus, the relatively small difference between the frequency of the optical source and the frequency of the millimeter-wave source suggests the feasibility of our approach. Consequently, the resistance $R_{g}$ simulates the losses due to the coupling between fiber and photodiode. Under these conditions, we want to estimate $R_{g}$ so that the speed of response of the photodiode would be at a maximum. In order to achieve this maximum, the effective quality factor must be at a minimum. Assuming a photodiode with an infinite reverse internal resistance, we can write the well-known formula

$\mathrm{Q}_{\mathrm{eff}}=\frac{\mathrm{Q}}{1+\frac{2 \pi f_{0} \mathrm{LQ}}{\mathrm{R}_{\mathrm{g}}}}$

where $Q_{\text {eff }}$ is the effective $Q$. 
By substituting Eqn. (4) and $Q=2 \pi f_{0} \mathrm{~L} / \mathrm{R}$ into Eqn. (7) we find

$\mathrm{Q}_{\mathrm{eff}}=\sqrt{\mathrm{LC}} \cdot \frac{\mathrm{R}_{\mathrm{g}}}{\mathrm{RCR}_{\mathrm{g}}+\mathrm{L}}$

By considering in Eqn. (8) $Q_{\text {eff }}$ as a function of $R_{g}$, it is easy to see that this function has no relative maxima and minima, but we deduce

$0 \leqslant \mathrm{Q}_{\mathrm{cff}}<\frac{1}{\mathrm{R}}\left(\frac{\mathrm{L}}{\mathrm{C}}\right)^{1 / 2}$

Maximum bandwidth corresponds to minimum quality factor. From Eqn. (9) it is derived that $\mathrm{Q}_{\mathrm{eff}}(\mathrm{min})$ is equal to zero, which corresponds to $\mathrm{R}_{\mathrm{g}}=0$. Conversely, for large losses we have a $-3 \mathrm{~dB}$ effective bandwidth near $(1 / \mathrm{R})(\mathrm{L} / \mathrm{C})^{1 / 2}$ (broadband circuit).

When $\mathrm{R}_{\mathrm{g}}$ is small, a first-order Mc. Laurin expansion of Eqn. (8) is valid, namely

$\mathrm{Q}_{\mathrm{eff}} \cong\left(\frac{\mathrm{C}}{\mathrm{L}}\right)^{1 / 2} \cdot \mathrm{R}_{\mathrm{g}}\left(1-\frac{\mathrm{RC}}{\mathrm{L}} \mathrm{R}_{\mathrm{g}}\right)$

which corresponds to an effective $-3 \mathrm{~dB}$ bandwidth given by

$\mathrm{B}_{\mathrm{eff}}=\frac{f_{0}}{\mathrm{Q}_{\mathrm{cff}}}=\frac{1}{2 \pi \mathrm{CR}_{\mathrm{g}}\left(1-\frac{\mathrm{RC}}{\mathrm{L}} \cdot \mathrm{R}_{\mathrm{g}}\right)}$

\section{DISCUSSION}

Several researchers studied III-V photodiodes in order to obtain conclusions on bandwidth. We refer to Wang and Bloom, Smith et al. ${ }^{6}$, Auston and Smith ${ }^{7}$, etc.

The procedure used in order to derive the expression for the $-3 \mathrm{~dB}$ effective bandwidth when $R_{g}$ is small is very useful since the value of this fictitious resistance can be obtained experimentally. Applying Eqn. (11), we find an optimum value for the $-3 \mathrm{~dB}$ effective bandwidth (broadband circuit). GaAs PIN photodiodes with high speed response can be characterized by using the method exposed previously.

In addition, we can claim that our results are in good agreement with bandwidth values obtained experimentally by various researchers ${ }^{8.9}$, but our theoretical procedure is new.

\section{REFERENCES}

1. C.H. Lee, Appl. Phys. Lett., 30, 2, pp. 84-86 (1977).

2. K. Wilson, GEC J. Res. 4, 2, pp. 140-146 (1986). 
3. S.Y. Wang and D.M. Bloom, Elect. Lett., 19, pp. 554-555 (1983).

4. S.Y. Wang, D.M. Bloom and D.M. Collins, Appl. Phys. Lett. 42, pp. 190-192 (1983).

5. R.W. Dixon and N.K. Dutta, ATT Tech. Journal, 66, 1, pp. 73-83 (1987).

6. P.R. Smith, R.P. Hooper and I. Garrett, Opt. and Quantum Elect. 10, pp. 293-300 (1978).

7. D.H. Auston and P.R. Smith, Appl. Phys. Lett., 41, pp. 595-601 (1982).

8. N.K. Dutta, ATT Tech. J., 68, 1, pp. 11-13 (1989).

9. J.M. Wiesenfeld, IBM J. Res. Dev., 34, 2/3, pp. 141-161 (1990). 

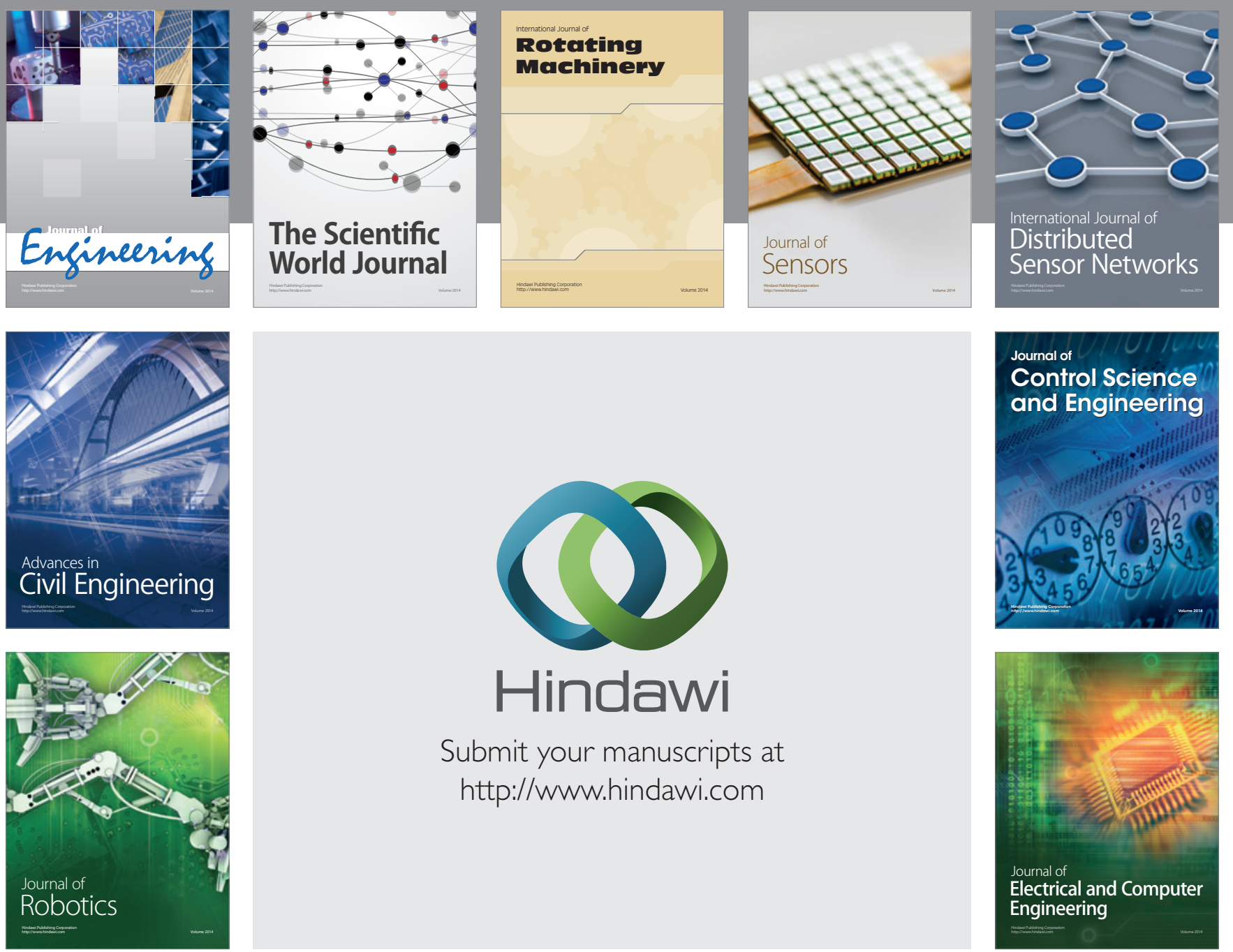

Submit your manuscripts at

http://www.hindawi.com
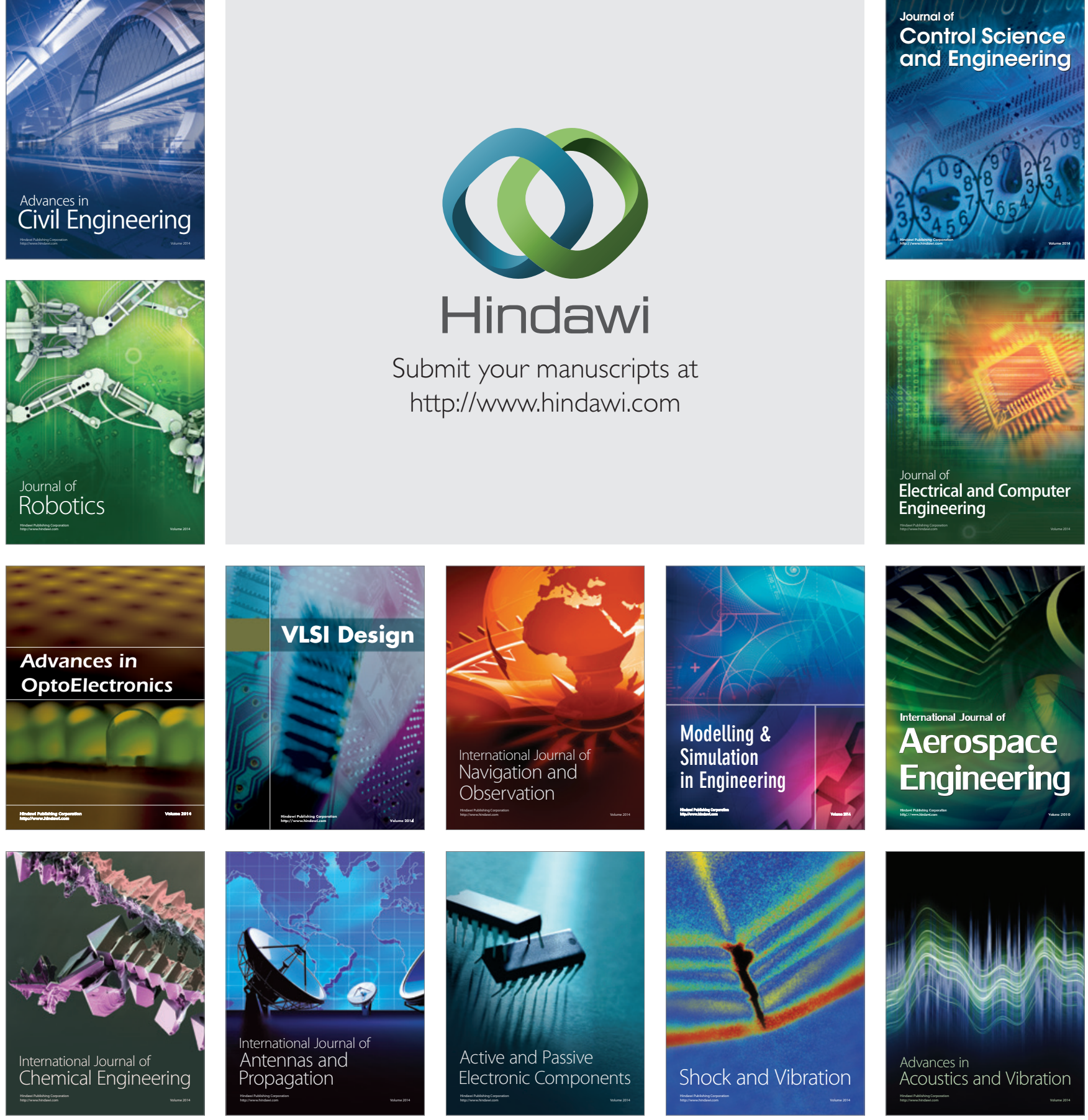